\title{
An Experience Report on Update of Systematic Literature Reviews
}

\author{
Lina Garcés \\ Dept. of Computer Systems \\ University of São Paulo \\ São Carlos, SP - Brazil \\ IRISA - University of South Brittany \\ Vannes, France \\ linamgr@icmc.usp.br
}

\author{
Katia R. Felizardo \\ Department of Computer \\ Federal Tech. Univ. of Paraná \\ Cornélio Procópio, PR - Brazil \\ katiascannavino@utfpr.edu.br
}

\author{
Lucas Bueno R. Oliveira Elisa Yumi Nakagawa \\ Federal Inst. of São Paulo Dept. of Computer Systems \\ São Carlos, SP - Brazil \\ University of São Paulo \\ São Carlos, SP - Brazil \\ elisa@icmc.usp.br
}

\begin{abstract}
Context: In order to preserve the value of Systematic Literature Reviews (SLRs), they should be frequently updated including new studies produced after the conduction of the reviews. However, most of SLRs are outdated and there is a lack of works that support the conduction of SLRs updates. Objective: The main goal of this paper is to report our experience in updating two of our SLRs. Method: To update these two SLRs, we used automated techniques based on VTM (Visual Text Mining) to guarantee the presence of relevant studies. Results: From our experience, some factors to satisfactorily update SLRs were identified: (i) to adopt software tools to support the updating process; (ii) to provide as much as possible information of previous SLR; (iii) to involve researchers from previous SLR; and (iv) to reuse protocol from preliminar SLR. Conclusions: Reported lessons learned can be used as a basis of knowledge to guide researchers when updating their SLRs.
\end{abstract}

Keywords-Systematic literature review, update, VTM

\section{INTRODUCTION}

Evidence Based Software Engineering (EBSE) was first introduced in 2004 as a means of advancing and improving the discipline of Software Engineering (SE) [1]. In this context, Systematic Literature Review (SLR) (a.k.a. Systematic Review (SR)) has provided a methodical, structured process to support the conduction of literature reviews [1] and has then gained substantial importance [2].

If a research area is continually evolving (as it is common in computing), SLRs that are not maintained (i.e., updated) can become out of date. Incorporation of new research or studies (i.e., evidence) into existing SLRs is therefore paramount to sustain their relevance. In other words, SLRs should be frequently updated with the purpose of identifying new evidence that has emerged after the completion of the reviews.

It is worth highlighting that, in the Medicine area, SLRs are in general updated at least every two years, determining whether or not there are new studies available for inclusion in the previous review [3]. In the SE area, the main reasons to update SLRs is that SE professionals and researchers may rely on the results of SLRs to build a body of knowledge about when, how, and what process, techniques, methods, tools, and

DOI reference number: 10.18293/SEKE2017-078 others are more appropriate to be used. Besides that, SLRs in the SE area have also contributed to identify new, important research topics that have not been treated, yet. Therefore, we argue that the update of SLRs is also a quite important issue in SE.

Even when the same authors update their reviews, search and selection tasks can consume considerable amount of time and efforts, especially if many new studies are obtained during the search, which can lead to difficulties in reading and evaluating evidences. Hence, it may be beneficial to have approaches, including techniques and tools, which support update of SLRs.

Considering that up-to-date SLRs are quite important, but effective update approaches have not been widely investigated in the literature, in our previous paper [4], we presented an automated approach based on Visual Text Mining (VTM) to support the update of SLRs. Such paper also describes an experiment to compare the outcomes achieved using this approach to the ones using traditional (manual) approach. The goal of this paper is to present an experience report on the use of our approach to update SLRs executed by SE researchers. The specific contributions of our work to the body of knowledge in EBSE field are:

1) we bring an experience report by analyzing two real SLRs updated by SE researchers;

2) we present the application of VTM techniques in real SLRs; and

3) we summarized a set of lessons that we have learned when updating SLRs and that could be useful in the context of other SLRs that are intended to be updated.

The remainder of this paper is organized as follows. Section II presents related works and a brief background on how VTM has been used for SLRs. Section III presents the report of our experience in updating SLRs. Section IV presents a brief discussion together with the lessons learned. Finally, conclusions are presented in Section V.

\section{BACKGROUND AND RELATED WORKS}

The process used to extract high-level knowledge from lowlevel data is known as Knowledge Discovery in Databases (KDD) [5]. Data Mining (DM) is a part of the KDD process 
responsible for extracting patterns or models from data. Visual Data Mining (VDM) is a combination of visualization and traditional DM techniques and is used to explore large datasets [5]. A specific application of VDM, which is of interest in our work, is the amalgamation of text processing algorithms with interactive visualizations to support users to make sense of text collections. By extension, Visual Text Mining (VTM) refers to VDM applied in text or to a collection of documents. According to Paulovich and Minghim [6], the use of VTM can speed up the process of interpreting and extracting useful information from document collections.

Four studies have investigated the use of VTM within the context of EBSE [4], [7], [8], [9].

Malheiros et al. [7] applied content-based VTM techniques to support the selection of primary studies. Similarly, the approach presented by Felizardo et al. [8] also used VTM techniques in the SLR process; however, this approach contains additional visualization techniques based on meta-data analysis. Both works [7], [8] compared the performance of reviewers in carrying out the selection of primary studies by reading abstracts or by using VTM techniques. The works concluded that VTM provides a more precise selection of relevant studies, speeding up the selection task.

Felizardo et al. [9] extended their previous work [8] to support the selection of primary studies, evaluating the decisions of including or excluding primary studies and, mainly, supporting reviewers to ensure as far as possible that important studies are not removed. They concluded that VTM can give solid clues about which particular studies should be checked, reducing the volume of documents that need to be re-evaluated and the time spent in the whole process. VTM therefore seems to be beneficial in supporting the SLR process.

Moreover, VTM has also been beneficial to update SLRs [4] and an approach, called USR-VTM, was proposed and will be also explored in the context of this paper. Because of this, a summary of this approach is presented in next section.

\section{A. USR-VTM Approach}

In order to update SLRs using USR-VTM , this approach receives a set of primary studies as input, including [4]: (i) studies included in the previous review; (ii) studies excluded in the previous review; and (iii) studies to be evaluated in the current review (i.e., the new evidence). In order to support selection of new primary studies, two visual representations are built: content-map and edge bundles ${ }^{1}$.

A content-map is a two-dimensional visual representation where each $m$-dimensional instance - a document (primary study) - is mapped on the screen as a graphic element, normally a circle. The process to create a content-map involves the conversion of all primary studies (title, abstract, and keywords) into multi-dimensional vectors. The dimensionality is based on all the terms extracted from the primary studies and it can be reduced eliminating stopwords (i.e., minimally representative terms, such as prepositions, articles, and conjunctions), applying stemming (i.e., converting terms to their radical; for

\footnotetext{
${ }^{1}$ In general, visualization techniques use colours to add extra information on a visual representation; hence, we recommend reading of a colour version of this paper.
}

instance, "testing" and "tester" are both reduced to "test") and using projection techniques [10]. In short, a content-map place studies (represented as circles) on the 2D layout in a way that reflects similarity relationships. Thus, similar studies are placed close to one another and dissimilar studies are positioned far apart.

Similarity is calculated using the cosine similarity measure, often used to compare documents in text mining. It is a measure of similarity between two vectors of $n$ dimensions by finding the cosine of the angle between them, which ranges between 0 (no similarity) and 1 (completely similar) [11]. The content-map presented in Figure 1 contains studies included (green points) and excluded (red points), in a previous SLR, as well as studies to be evaluated (grey points) in the update of such review. Primary studies are connected with their neighbours by applying KNN (K-Nearest Neighbour) Edges Connection technique [12]. This technique connects nodes with their nearest neighbours, computing the proximity on the projection itself.

Another visual representation is edge bundles, which is a hierarchical tree visualization technique that shows both nodes and node-links (relationships between nodes) [13]. In the case of an SLR, nodes (small circles, as shown in Figure 1) are the primary studies (i.e., studies included in the previous review, studies excluded in the previous review, and studies to be evaluated in the current review) and node-links (blue lines, also shown in Figure 1), are the citations between them.

In order to create edge bundles, the HiPP (Hierarchical Point Placement) strategy [6] is used. In the edge bundles view, node-links were coloured to represent the direction of the citation: the citing study is at the light blue end of the link and the cited study at the dark blue end. In summary, analyzing the edge bundles it is possible to identify the number of times that a study has been cited by other studies. Notice that studies represented in the content-map are the same ones in the edge bundles; i.e., each study in the content-map has a corresponding study in the edge bundles.

Revis $^{2}$ tool was used to create these visual representations. It takes only seconds to create and present these views with a few hundred documents.

Two strategies to include and exclude new primary studies using both content-map and edge bundles can be applied to support selection of studies to update SLRs [4]:

- Inclusion Strategy: To include a new primary study, it must be a neighbour of at least one previously included study (observed in the content-map) AND it must not cite previously excluded paper(s) (observed in the edge bundles). Neighbours are nodes connected through edges between them; and

- Exclusion Strategy: To exclude a primary study, it must be neighbour of only previously excluded studies (or studies in evaluation) AND it must not cite previously included papers.

\footnotetext{
${ }^{2}$ Revis is a visualization and interaction tool that offers Visual Text Mining exploration of document collections. It is freely available at https://www. dropbox.com/sh/2ahjt6urucm114s/AAD8GF3WmNAtYrvHbzOKzgeqa?dl=0
} 
Undefined or unclear situations should be given particular attention. For example, a study may be a neighbour of previously excluded studies; however, it cites previously included study(ies), i.e., the New Evidence - NE (grey points) are linked to previously excluded study(ies) (red point(s) in the contentmap) and cite previously included study(ies) (green point(s) in the edge bundles). In a situation such as this, the new primary study should be analyzed by reviewers.

By exploiting the strong visual processing abilities of humans, the USR-VTM approach is an important ally to be used during update of SLRs, facilitating and enhancing interpretation and decision-making in regard to the selection of new primary studies. USR-VTM can give solid clues (except to undefined situations) about which particular studies should be included or excluded. However, the final decision is taken by researchers (experts in the SLR domain). In the following, we present our experiences in updating two SLRs using the two strategies described bellow for selection of new studies.

\section{EXPERIENCE REPORT}

This section presents our experiences in updating two SLRs. The first one (hereafter referred to as SLR1) had the goal of finding the state of the art about Reference Architectures and Reference Models for Ambient Assisted Living (AAL) [14]. The second one (referred to as SLR2) was focused on works about development of service-oriented robotic systems [15]. It is observed that the first version of both SLR1 and SLR2 had been conducted at least two year ago, then an update becomes necessary. In next sections, we discuss our experiences in updating both SLRs (referred as SLR1' and SLR2').

\section{A. Updating SLR on Reference Architectures and Reference Models in $A A L$}

To update SLR1, we revisited its protocol that contains all required information to execute an SLR (e.g., research questions, search string, search databases, among others). It is worth saying that only the period to be updated was changed. Following, we conducted the searches in the same databases used in SLR1 (namely, ACM Digital Library, IEEE xplore, Springer, ScienceDirect, Compendex, Scopus, and Web of Science) and also using the same search string. All primary studies were managed using $\mathrm{JabRef}^{3}$, which is an open source bibliography reference manager tool. We exported information of all primary studies found (i.e., title, author(s), abstract, keywords, year of publication, and the name of the data source) to JabRef. For databases that do not support JabRef, we manually get such information. During the searches, we considered studies published after November, 2015. We needed to remove repeated studies, as different databases can sometimes find the same studies. As a result, a total of 84 non-repeated studies were obtained.

Aiming to select the primary studies to be in fact included in SLR1', two researchers performed independently a brief reading only considering titles and abstracts of the studies, intending to exclude studies far away from the topic of this SLR. A total of 23 studies (27.4\% of 84 studies) were considered to be further analyzed. After that, we used the

\footnotetext{
${ }^{3}$ http://www.jabref.org/
}

techniques based on VTM described in Section II-A. Hence, we applied USR-VTM that generated content-map and edge bundles for this update, as showed in Figure 1. As mentioned previously, the 14 studies included in SLR1 are coloured in green, the 50 studies excluded in SLR1 are coloured in red, and the 23 studies to be analyzed are coloured in grey. USRVTM was then applied to analyze the 23 new studies. For sake of space limitation, Figure 1 exemplifies three of them (NE[17], NE[18], and NE[20]).

As suggested by the Inclusion Strategy of USR-VTM, $\mathrm{NE}[17]$ is neighbour of at least one previously included study (green points in the content-map) and it do not cite previously excluded papers (red points in the edge bundles). When an $\mathrm{NE}$ is allocated in the content-map next to other included papers, there is a strong indication that this NE should be included. It is also possible to observe that NE[17] indeed do not share citations with excluded papers (red points). In both views (content-map and edge bundles), we had clues that NE[17] is relevant for our review and that it should be included. Therefore, our final decision was to include it.

The second study is NE[18] (pointed out in Figure 1), which is neighbour of previously excluded studies; however, it cites previously included studies. NE[18] was classified as an undefined situation by USR-VTM. In this case, this study was read by us and the final decision was to excluded it. This study deserved special attention.

The third study is NE[20] (pointed out in Figure 1). As suggested by the Exclusion Strategy of USR-VTM, NE[20] is neighbour of previously excluded studies (red points in the content-map) and it is not linked to previously included papers (green points in the edge bundles). On the other hand, $\mathrm{NE}[20]$ is linked to one previously excluded paper. Therefore, the views encouraged us to exclude NE[20].

After applying the Inclusion/Exclusion Strategies in all 23 studies, including those ones exemplified above, we selected seven new studies to be included, resulting in a total of 21 relevant studies for this SLR (14 from SLR1 plus seven new studies). After that, the full text of these seven studies was read and the required data for updating the answers of the research questions were extracted. For this, the data extraction form created to SLR1 was used without changes. For each new included study, this form was filled by one researcher. For validation purposes, a sample comprising $30 \%$ of the total number of primary studies was selected randomly and had their data extracted by other researchers. Whenever the data extracted differed, differences was discussed until consensus was reached. The data synthesis involved the compilation of the data extracted from each new primary study and merge of such data into the previous answers for each research question. Besides that, in the previous version of this SLR, the data synthesis for the research questions was performed using a quantitative table. Reuse of same table facilitated the analysis during update. Finally, it is also worth highlighting that SLR1 and SLR1' were conducted by the same researchers.

\section{B. Updating SLR on Robotics}

To update SLR2, initially, its protocol was revisited to identify required changes. The research questions were not changed, but the search strategy was modified to restrict the 


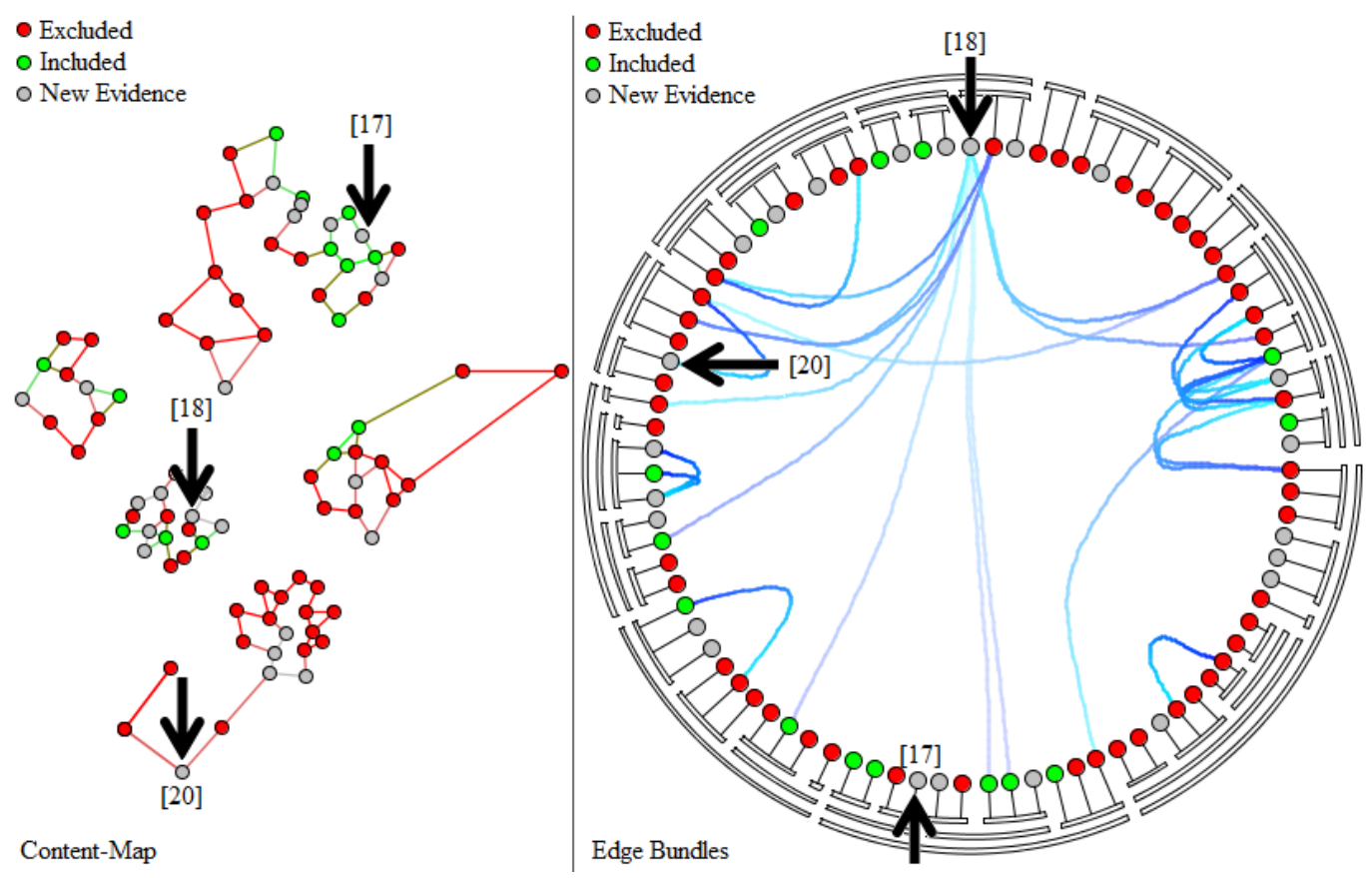

Fig. 1. Views to support the update of SLR1.

search only for studies published after 2011, avoiding overlap among studies retrieved in SLR2 and SLR2'.

To find new primary studies, we used the same databases used in SLR2. New terms to the search string were not identified, but minor changes were required because during 2014, some search engines were modified, demanding adaptations of search strings. After changing the protocol, it was tested to verify its feasibility and adequacy based on a pre-selected set of relevant studies. A total of 158 studies were retrieved from the search engines. Following, repetitive studies were removed and after a brief reading in the title and abstract of each study, we selected 28 studies that could be relevant to this SLR.

Similarly to SLR1', the study selection activity of SLR2' was performed with the support of USR-VTM and its views. Figure 2 shows the content-map and edge bundles related to this SLR. As mentioned previously, in these views, the 39 studies included in SLR2 are coloured in green, the 36 studies excluded in SLR2 are coloured in red, and the 28 studies to be analyzed are coloured in grey. These views were generated in the same way described in Section III-A. In the sequence, we illustrate the application of USR-VTM in three of the 28 studies (NE[5], NE[14], and NE[26]).

$\mathrm{NE}$ [5] (pointed out in Figure 2) is the first example. As suggested by Inclusion Strategy of USR-VTM, NE[5] is neighbour of studies in evaluation) and it must not cite previously included papers. Consequently, we decided to exclude it.

NE[14] (pointed out in Figure 2) is neighbour of at least one previously included study and it does not cite previously excluded papers. On the other hand, NE[14] cites four previously included papers. This fact might be taken as a strong indication of $\mathrm{NE}[14]$ is a relevant study, indicating its inclusion. Therefore, our final decision was to include it.

$\mathrm{NE}[26]$ (pointed out in Figure 2) is a typical example of a study difficult to be selected, because it is neighbour of previously excluded studies and it cites three previously included studies. This study was carefully analyzed by us. We decided by its inclusion. As a result, 18 primary studies were then selected for full reading and data extraction.

With the final set of primary studies decided upon, the data extraction activity was carried out on all 18 studies that passed the screening process. The information for answering each research question was tabulated using themes, which were defined by one researcher. Thereafter, studies belonging to each theme were counted. This researcher was also responsible to extract the data and complete the data extraction form. Results were summarized to present an overview of the findings. The data synthesis were performed using tables (showing the totals and summaries).

\section{DISCUSSIONS}

The two case studies presented herein were carried out aiming to investigate and evaluate the use of USR-VTM for updating real SLRs involving SE researchers. The main advantage of USR-VTM is that the selection activity (classification of studies as included or excluded) is based on previous knowledge. For example, if a study was considered relevant (i.e., it was included) in a previous SLR, and a new study is similar in terms of content to this study, then it is a clue that the new study could be included. Similarly, if a new study cites or is cited by previous relevant studies, then it could be included. On the other hand, a new study that is dissimilar in terms of content of all previous included studies can probably be excluded. In addition, a new study that cites or is cited by previous excluded studies has a chance to be irrelevant to the scope of the systematic review. As USR-VTM is new in EBSE field, for a while, it is not our intention to recommend the elimination of the traditional selection approach 

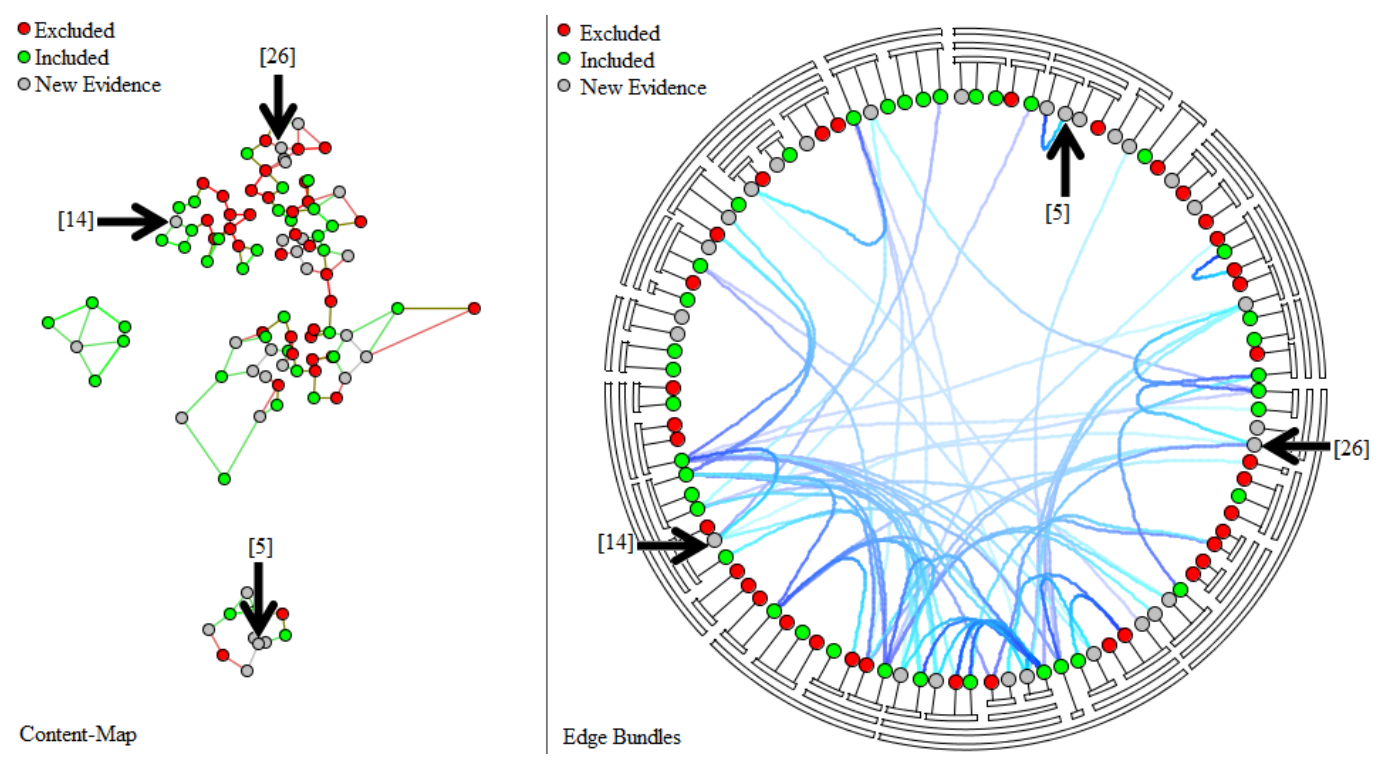

Fig. 2. Views to support the update of SLR2.

(title/abstracts/full text reading). USR-VTM intends to be a complementary approach that can be used in conjunction with the traditional one, mainly to speed up the selection activity. In this perspective, USR-VTM could be also used before or after applying the traditional approach for selecting new primary studies, aiming to be a double-checking mechanism.

Considering our experience with regard to both updates and other SLRs that we have updated in the last years, following, we discuss four main lessons learned:

- Lesson 1 - Choose tools to support updates: When researchers are conducting an SLR, selection of relevant studies can consume considerable time and effort. In particular, the obtaining of many studies during the search task leads to difficulties in reading and evaluating the set of studies. To update SLRs, adopting a supporting software tools is also quite important to mainly facilitate primary studies management during the selection of new studies. They become even more important when they store primary studies found in the previous version of the SLR and information of these studies will be used to update. When it is intended to apply an approach like USR-VTM[4], supporting tooling together with data of primary studies (e.g., LaTeX . bib files) are essential. We adopted Jabref tool to support both conduction and update of SLRs.

- Lesson 2 - Keep information of previous SLRs: If it is intended to conduct an update of an SLR, it is essential to keep all information related to that SLR, especially the set of studies returned from each database, the adapted search string for each database, and even the set of excluded studies. In particular, as presented in our experiences in this paper, when approaches like USR-VTM are intended to be used during update, previously studies included and excluded are required (i.e., it is necessary to register and make available the.$b i b$ files containing both set of studies). Furthermore, previous information is a rich source for future checking. For example, it can answer doubts, such as which were the reasons to a given study to have been included/excluded and who included/excluded it, why those databases were selected and used, why some terms were not considered in the search string, and so on. While this lesson is of course valuable, we claim that it is usually underrated by EBSE researchers. We recommend to EBSE researchers that they make available such type of information on their SLRs, instead of only the final report.

- Lesson 3 - Form a team containing a researcher who has already participated in the previous review: We argue that the effort to update an SLR is significantly higher if none of the researchers of the previous review as part of the team, providing direct access to the employed instruments. In spite of the SLR documentation should make it possible to be updated by any team of researchers, there is a tacit knowledge (in the mind of researchers) that the documentation related to SLRs do not mention. There is an amount of implicit knowledge (that we refer as "global scenario" of the research topic of the SLR) acquired from a large number of primary studies usually found in the previous execution of SLRs. It is interesting to say that this global scenario contains knowledge from both included and excluded studies, as well as the entire process and tasks by which the SLR was carried out. Therefore, the direct gain using the same (or part of the same) team is take advantages of the global scenario in the mind of researchers. There is therefore a faster access to information (even documented information), while new teams also need to understand how the information is organized.

For instance, considering our experience regarding SLR1/SLR1', the global scenario was formed from 273 studies analyzed in the first conduction. As we used the same team during update, this global scenario was fundamental to analyze other 84 studies during 
update. Otherwise, if new teams updated it, these 84 studies needed to be analyzed without any support of previous acquired knowledge.

It is worth also highlighting that there is a lack of concrete studies proposing or discussing about the ideal researchers team (in terms of number of researchers and their skills) for both SLR conduction and update. However, from our experiences in conducting and updating SLRs, teams should include at least three researchers: two experts in the research topic being explored in the SLR (at least one being a high experienced researcher) and one specialist in SLR. It is also important that all team members are familiar with conduction of SLRs and have experience in using supporting tools required for SLRs, e.g., Revis and JabRef.

- $\quad$ Lesson 4 - Reuse SLR protocol: As well known, the establishment of the SLR protocol usually consumes a considerable amount of time and effort, besides being a complex task. However, it is essential to assure the quality of SLRs. Reusing such protocol is undoubtedly interesting when updating SLRs. In our two experiences, We replicated the original protocols. In this context, we experienced the importance of having access to a complete and detailed protocol to guide our updates. The time spent in checking SLR protocols was quite reduced if compared to protocol preparation to the first conductions. Only considering our two SLRs, the time consumed to check the protocol was less than $1 / 5$ of the time consumed to prepare a protocol from scratch. Therefore, reusing the protocol is in fact quite relevant.

\section{COnClusions And Future Work}

Since knowledge in any research area continually evolves, SLRs that intend to represent such knowledge must be also from time to time updated. Otherwise, their validity can be undetermined. In this scenario, when SLRs are updated, considerable efforts must be applied; besides, no guidelines on how to conduct such updates are available. The main contribution of this work was to report experiences in updating two SLRs and also report lessons learned, thus providing a basis for other researchers who intend to update SRLs.

We also showed that the use of USR-VTM approach can support and speed up the SLR updates. This work has helped us to understand the usefulness of VTM techniques to support those updates. We observed that USR-VTM can support tasks that involve large collections of data, such as the studies collected and to be evaluated. Through these two experiences and others, we argue that adoption of supporting tools, like that one automating USR-VTM, brings important advantages, mainly facilitating the selection of new studies and also becoming results of SLR updates more trustworthy. In a scenario of a team of reviewers conducting an SLR, as the case of SLR1' and SLR2', USR-VTM can be considered a valuable means to reach the conclusion on what should and should not be included. The views supported the team to reach a common sense about inclusions and exclusions. On the other hand, in the special case of an SLR executed by only one reviewer, this researcher should consider discussing his or her decisions with other researchers. Alternatively, the researcher could use our approach as the external opinion. Moreover, by exploring different visual representations of primary studies through USR-VTM' views, researchers have additional and complementary detailed information, for example, citations between primary studies, which is not readily available only reading studies.

For future work, we intend to use USR-VTM to also update Systematic Mappings (SM), considering that selection of new primary studies is quite similar in both SM and SLR; therefore, we believe our approach is also suitable to it.

Acknowledgments This work is supported by FAPESP (Grants N.: 2013/20317-9, 2014/02244-7, and 2015/19192-2).

\section{REFERENCES}

[1] B. Kitchenham and S. Charters, "Guidelines for performing systematic literature reviews in software engineering," Keele University and Durham University, UK, Tech. Rep. EBSE 2007-001, 2007.

[2] H. Zhang, B. Muhammad, and T. Paolo, "Identifying relevant studies in software engineering," Information and Software Technology, vol. 53, no. 6, pp. 625-637, 2011.

[3] J. Higgins, S. Green, and R. Scholten, Maintaining Reviews: Updates, Amendments and Feedback, 2008, pp. 31-49.

[4] E. Felizardo, K.R. ad Nakagawa, S. MacDonell, and J. Maldonado, "A visual analysis approach to update systematic reviews," in $18^{t h}$ Int. Conference on Evaluation and Assessment in Software Engineering (EASE'14), 2014, pp. 4:1-4:10.

[5] D. Keim, "Information visualization and visual data mining," IEEE Transactions on Visualization and Computer Graphics, vol. 8, no. 1, pp. 1-8, 2002.

[6] F. Paulovich and R. Minghim, "Hipp: A novel hierarchical point placement strategy and its application to the exploration of document collections," IEEE Transactions on Visualization and Computer Graphics, vol. 14, no. 6, pp. 1229-1236, 2008.

[7] V. Malheiros, E. Höhn, R. Pinho, M. Mendonça, and J. Maldonado, "A visual text mining approach for systematic reviews," in $1^{\text {st }}$ Int. Symposium on Empirical Software Engineering and Measurement (ESEM'07), 2007, pp. 245-254.

[8] K. Felizardo, N. Salleh, R. Martins, E. Mendes, S. MacDonell, and J. Maldonado, "Using visual text mining to support the study selection activity in systematic literature reviews," in $5^{\text {th }}$ Int. Symposium on Empirical Software Engineering and Measurement (ESEM'11), 2011, pp. $1-10$.

[9] K. Felizardo, G. Andery, F. Paulovich, R. Minghim, and J. Maldonado, "A visual analysis approach to validate the selection review of primary studies in systematic reviews," Information and Software Technology, vol. 54, no. 10, pp. 1079-1091, 2012.

[10] F. V. Paulovich, L. G. Nonato, R. Minghim, and H. Levkowitz, "Least squares projection: a fast high precision multidimensional projection technique and its application to document mapping," IEEE Transactions on Visualization and Computer Graphics, vol. 4, no. 3, pp. 364-375, 2008.

[11] P. Tan, M. Steinbach, and V. Kumar, Introduction to Data Mining, 1st ed. Pearson, 2014.

[12] N. S. Altman, "An introduction to kernel and nearest-neighbor nonparametric regression," The American Statistician, vol. 46, no. 3, pp. 175-185, 1992.

[13] D. Holten, "Hierarchical edge bundles: Visualization of adjacency relations in hierarchical data," IEEE Transactions on Visualization and Computer Graphics, vol. 12, no. 5, pp. 741-748, 2006.

[14] L. Garcés, A. Ampatzoglou, F. Oquendo, and E. Y. Nakagawa, "Reference architectures and reference models for ambient assisted living systems: A systematic literature review," University of São Paulo (USP), Tech. Rep. No. 414. 1-32, 2017.

[15] L. B. R. Oliveira, "Architectural design of service-oriented robotic systems," Ph.D. dissertation, University of São Paulo (USP) / Université de Bretagne-Sud (UBS), São Carlos, Brazil / Vannes, France, 2015. 